\title{
Determining the nucleation rate from the dimer growth probability
}

\author{
Joop H. ter Horst ${ }^{\mathrm{a})}$ \\ Laboratory for Process Equipment, Delft University of Technology, Leeghwaterstraat 44, 2628 CA Delft, \\ The Netherlands \\ Dimo Kashchiev \\ Institute of Physical Chemistry, Bulgarian Academy of Sciences, ul. Acad. G. Bonchev 11, \\ Sofia 1113, Bulgaria
}

(Received 10 June 2005; accepted 27 July 2005; published online 22 September 2005)

\begin{abstract}
A new method is proposed for the determination of the stationary one-component nucleation rate $J$ with the help of data for the growth probability $P_{2}$ of a dimer which is the smallest cluster of the nucleating phase. The method is based on an exact formula relating $J$ and $P_{2}$, and is readily applicable to computer simulations of nucleation. Using the method, the dependence of $J$ on the supersaturation $s$ is determined by kinetic Monte Carlo simulations of two-dimensional (2D) nucleation of monolayers on the (100) face of Kossel crystal. The change of $J$ over nearly 11 orders of magnitude is followed and it is found that the classical nucleation theory overestimates the simulation $J$ values by an $s$-dependent factor. The 2D nucleus size evaluated via the nucleation theorem is described satisfactorily by the classical Gibbs-Thomson equation and its corrected version accounting for the spinodal limit of 2D nucleation. (C) 2005 American Institute of Physics.
\end{abstract}

[DOI: $10.1063 / 1.2039076]$

\section{INTRODUCTION}

The determination of the stationary nucleation rate $J$ is a central problem in nucleation theory and experiment. ${ }^{1,2}$ This quantity gives the number of new-phase clusters with large enough size that are steadily nucleated per unit time and unit volume (or area) of the supersaturated old phase. Experimentally, the determination of $J$ is not easy because of the difficulties in the realization of time-independent supersaturation $\Delta \mu$ and of an old phase with well-defined properties (e.g., purity, number and activity of nucleation-active centers, etc.). Such difficulties do not exist in computer simulations of nucleation and for that reason these are valuable tools for deeper understanding of the nucleation process at the molecular level and for reliable verification of theoretical expressions for the nucleation rate.

However, the direct determination of $J$ by computer simulations is also accompanied with difficulties. A major problem is the excessively long computer time required by the presently available methods to obtain $J$ when the nucleating system is subjected to the relatively low supersaturations used in real experiments. As the computer time for finding $J$ shortens strongly with increasing $\Delta \mu$, these methods are only applicable to systems at high enough supersaturations in a rather narrow range. There exists thus a necessity of new methods for direct determination of $J$, which would allow the simulations to be carried out within a reasonably short computer time, but for supersaturations in an extended range comprising relatively low $\Delta \mu$ values.

The objective of the present paper is to propose a new method for direct determination of the stationary nucleation

\footnotetext{
${ }^{a)}$ Author to whom correspondence should be addressed. Electronic mail: J.H.terHorst@wbmt.tudelft.nl
}

rate by computer simulations of the growth probability of a dimer which is the smallest cluster that can decay to a single molecule (or atom), i.e., to a monomer. In some cases this method may allow obtaining the $J(\Delta \mu)$ dependence for such low supersaturations that are comparable with those used in real experiments.

\section{THEORY}

Nucleation is known to occur by the formation and growth of $n$-sized clusters of the new phase ( $n$ is the number of molecules constituting the cluster). According to the Szilard model of nucleation, the clusters change their size by successive attachment and detachment of monomers to and from them. Within this model, the stationary nucleation rate $J\left(\mathrm{~m}^{-3} \mathrm{~s}^{-1}\right.$ or $\left.\mathrm{m}^{-2} \mathrm{~s}^{-1}\right)$ is given by the Becker-Döring formula ${ }^{1-3}$

$$
J=f_{1} C_{1} /\left(1+S_{2}\right),
$$

which is exact and applies to any kind of one-component nucleation [homogeneous, heterogeneous, three-dimensional (3D), two-dimensional (2D), etc.]. In Eq. (1) $f_{1}\left(\mathrm{~s}^{-1}\right)$ is the frequency of monomer attachment to a monomer, and $C_{1}\left(\mathrm{~m}^{-3}\right.$ or $\left.\mathrm{m}^{-2}\right)$ is the concentration of monomers. For instance, in one-component homogeneous nucleation of droplets or crystals in a vapor $C_{1}\left(\mathrm{~m}^{-3}\right)$ is the actual concentration of molecules in the vapor, and in $2 \mathrm{D}$ nucleation of liquid or crystalline monolayers on a solid substrate without nucleation-active centers on it $C_{1}\left(\mathrm{~m}^{-2}\right)$ is the actual concentration of adsorbed molecules on the substrate. The quantity $S_{2}$ is defined by 


$$
S_{2}=\sum_{n=2}^{M-1} g_{2} g_{3} \ldots g_{n} / f_{2} f_{3} \ldots f_{n}
$$

where $f_{n}\left(\mathrm{~s}^{-1}\right)$ and $g_{n}\left(\mathrm{~s}^{-1}\right)$ are, respectively, the frequencies of monomer attachment to and detachment from an $n$-sized cluster, and $M$ is the total number of molecules in the old phase.

On the other hand, the attachment and detachment frequencies govern the growth of an $n$-sized cluster to a macroscopically large cluster and the decay of the cluster to a monomer. Within the Szilard model, the corresponding growth and decay probabilities can be calculated ${ }^{4,5}$ with the help of a result from probability theory. ${ }^{6}$ The growth probability $P_{n}$ is given by the expression ${ }^{5}(2 \leqslant n \leqslant M-1)$

$$
P_{n}=1-S_{n} /\left(1+S_{2}\right),
$$

which is exact and valid for any kind of one-component nucleation and in which

$$
S_{n}=\sum_{i=n}^{M-1} g_{2} g_{3} \ldots g_{i} / f_{2} f_{3} \ldots f_{i} .
$$

While $P_{n}$ is not connected simply with $J$, the probability $P_{2}$ of growth of a dimer to a cluster with macroscopic size is. Indeed, at $n=2$ Eq. (3) yields

$$
P_{2}=1 /\left(1+S_{2}\right) .
$$

Combining Eqs. (1) and (5), we arrive at the following general relation between the stationary nucleation rate and the dimer growth probability:

$$
J=f_{1} C_{1} P_{2} .
$$

Within the Szilard model, this equation is also exact and applicable to any kind of one-component nucleation. It is a particular case of a more general equation found by White ${ }^{4}$ to relate $J$ and $P_{n}$.

Equation (6) has a very simple physical meaning. It says that $J$ is merely the product of the frequency $f_{1} C_{1}$ with which monomers become dimers and the probability $P_{2}$ that these dimers grow to microscopically large clusters rather than decay to monomers. This equation is very convenient for use in computer simulations, because then the monomer-tomonomer attachment frequency $f_{1}$ and the monomer concentration $C_{1}$ are either known independently or obtainable by separate simulations. Thus, the only quantity that has actually to be found by simulations is the dimer growth probability $P_{2}$. This can be done for a rather wide supersaturation range including $\Delta \mu$ values that in some cases may be as small as those used in real experiments. Moreover, employing $P_{2}$ for determination of $J$ with the help of Eq. (6) is advantageous, since the value of $P_{2}$ obtained by simulations is invariant with respect to the definition chosen for the clusters of size $n=3,4, \ldots$. This is so, because the simulations follow the evolution of a single dimer to a large enough cluster capable of irreversible growth irrespectively of how its size is defined.

When the dependence of $P_{2}$ on $\Delta \mu$ is obtained at constant temperature, it can be used for a model-independent determination of the nucleus size (the nucleus is that particu- lar cluster that requires maximum work for its formation). This can be done with the aid of the nucleation theorem. ${ }^{2,7-11}$ Indeed, representing $J$ in its general form ${ }^{1,2,12}$

$$
J=A \exp \left(-W^{*} / k T\right),
$$

from Eq. (6) we get

$$
P_{2}=\left(A / f_{1} C_{1}\right) \exp \left(-W^{*} / k T\right),
$$

where $A\left(\mathrm{~m}^{-3} \mathrm{~s}^{-1}\right.$ or $\left.\mathrm{m}^{-2} \mathrm{~s}^{-1}\right)$ is a kinetic factor, $W^{*}$ is the work to form the nucleus, $T$ is the absolute temperature, and $k$ is the Boltzmann constant. Taking the logarithm of both sides of Eq. (8) and employing the nucleation theorem (for one-component nucleation at constant $T$ ) in the form ${ }^{2,7,8,11}$

$$
d W^{*} / d \mu_{\text {old }}=-\Delta n^{*}
$$

results in

$$
\Delta n^{*}=k T d\left(\ln P_{2}\right) / d \mu_{\mathrm{old}}-k T d\left[\ln \left(A / f_{1} C_{1}\right)\right] / d \mu_{\mathrm{old}},
$$

where $\mu_{\text {old }}$ is the chemical potential of the old phase, and $\Delta n^{*}$, the excess number of molecules in the nucleus, is the difference between the number of molecules in the spatial region occupied by the nucleus and the number of molecules in the same region before the nucleus formed. When $\mu_{\text {old }}$ is changed at constant $T$, the quantities $A, f_{1}$, and $C_{1}$ are given by $^{2} \quad A=A_{0} \exp \left(\mu_{\text {old }} / k T\right), \quad f_{1}=f_{1,0} \exp \left(\mu_{\text {old }} / k T\right), \quad$ and $C_{1}$ $=C_{1,0} \exp \left(\mu_{\text {old }} / k T\right)$, where the factors $A_{0}, f_{1,0}$, and $C_{1,0}$ may depend only weakly on $\mu_{\text {old }}$. Hence, to a good approximation, Eq. (10) can be used in the form

$$
\Delta n^{*}=k T d\left(\ln P_{2}\right) / d \mu_{\mathrm{old}}+1 .
$$

For example, in the case of one-component nucleation of droplets or crystals in vapors behaving as ideal gas, we have ${ }^{1,2} \mu_{\text {old }}=\mu_{\text {ref }}+k T \ln p$, where $\mu_{\text {ref }}$ is a reference chemical potential and $p$ is the pressure of the vapors. Equation (11) then becomes

$$
\Delta n^{*}=d\left(\ln P_{2}\right) / d(\ln p)+1 .
$$

\section{SIMULATION RESULTS}

The kinetic Monte Carlo simulation results presented below are for one-component 2D nucleation of monolayers on the molecularly smooth surface of the (100) face of a Kossel crystal. The simulation details to determine the growth probability $P_{n}$ of an $n$-sized 2D cluster of monolayer thickness can be found elsewhere. ${ }^{13}$ The attachment frequency $k_{a}\left(\mathrm{~s}^{-1}\right)$ of a molecule to whatever site on the crystal surface is given by ${ }^{13-15}$

$$
k_{a}=k_{e} e^{s},
$$

where the dimensionless supersaturation $s$ is defined by

$$
s=\Delta \mu / k T=\left(\mu_{\mathrm{old}}-\mu_{e}\right) / k T,
$$

and $k_{e}\left(\mathrm{~s}^{-1}\right)$ and $\mu_{e}$ are the values of $k_{a}$ and $\mu_{\text {old }}$ at phase equilibrium $(s=0)$. As only nearest-neighbor interactions are taken into account, the detachment frequency $k_{d, j}\left(\mathrm{~s}^{-1}\right)$ of a molecule from a site with $j=0,1,2,3,4$ lateral nearest neighbors is of the form ${ }^{13-15}$ 


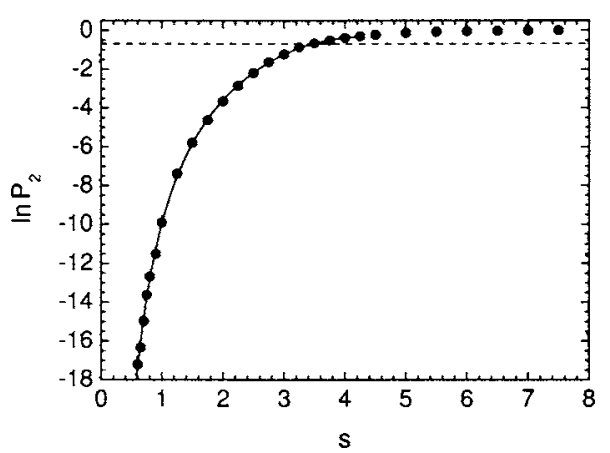

FIG. 1. Supersaturation dependence of the dimer growth probability: circles-simulation data; dashed line-the value $1 / 2$ of the probability; solid curve-Eq. (29).

$$
k_{d, j}=k_{e} e^{2 \omega(2-j)},
$$

where $\omega=\phi / k T$ is half the dimensionless overall nearestneighbor binding energy ( $\phi$ is half the value of this overall energy). The simulations were performed at a fixed $\omega=2$, so that the system studied is equivalent to the $2 \mathrm{D}$ Ising model with a nearest-neighbor coupling equal to $k T$. The supersaturation values used were in the range of $0.6 \leqslant s \leqslant 7.5$. A simulation run at a given value of $s$ started with a single dimer on the crystal face and ended either when the dimer decayed to a monomer (the run was then qualified as negative) or when the dimer grew to a cluster of sufficiently large size $n_{\text {max }}$ (the run was then qualified as positive). The dimer growth probability $P_{2}$ was calculated from the ratio of the number of positive runs to the total (positive and negative) number of runs. The total number of runs to obtain $P_{2}$ at a given supersaturation was between about $5 \times 10^{3}$ at the largest $s$ value and $4 \times 10^{8}$ at the smallest $s$ value. The maximum cluster size $n_{\max }$ for dimer growth was set equal to 200 , because it was found that clusters of this size practically did not decay to monomers.

In order to determine the nucleation rate $J$ with the help of Eq. (6) we need to know $f_{1}$ and $C_{1}$. In view of Eq. (13), the monomer-to-monomer attachment frequency $f_{1}$ is given exactly by

$$
f_{1}=4 k_{a}=4 k_{e} e^{s},
$$

because the monomer has four nearest-neighbor attachment sites at which a dimer can be created. The concentration $C_{1}$ of monomers on the crystal face can be evaluated from the approximate expression $^{14}$

$$
C_{1}=\left(1 / a_{0}\right) e^{s-4 \omega},
$$

which follows from Eqs. (13) and (15), since according to adsorption theory $C_{1}$ is approximately the product of the incidence rate $k_{a} / a_{0}$ of molecules and the average lifetime $1 / k_{d, 0}$ of an adsorbed molecule ( $a_{0}$ is the area occupied by such molecule on the crystal face). Combining Eqs. (6), (16), and (17) leads to the formula

$$
J(s)=4\left(k_{e} / a_{0}\right) e^{2 s-4 \omega} P_{2}(s)
$$

in which only the $P_{2}(s)$ dependence has to be obtained by simulations.

The circles in Fig. 1 represent $P_{2}$ as a function of $s$ in the

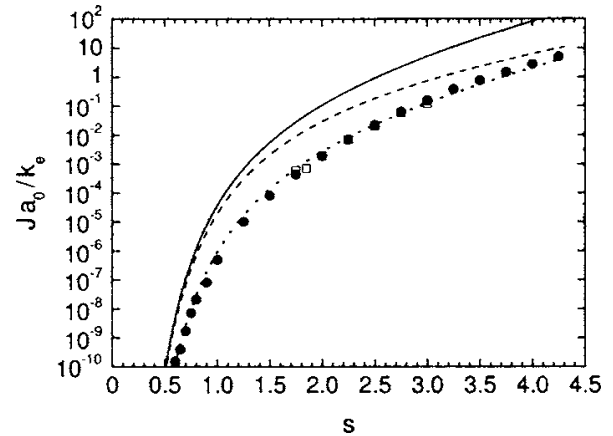

FIG. 2. Supersaturation dependence of the 2D nucleation rate: circlessimulation data obtained from Eq. (18) with the help of the $P_{2}(s)$ data in Fig. 1 ; squares - simulation data of Weeks and Gilmer; ${ }^{15}$ dashed curve-Eq. (25) of the classical nucleation theory; solid curve-Eq. (27); dotted curve-Eq. (27) with right-hand side multiplied by $1 / 40$.

supersaturation range studied. The standard deviation of the $P_{2}$ values is within the size of the circles. The dashed line visualizes the value $1 / 2$ of $P_{2}$. As seen, $P_{2}>1 / 2$ for $s$ $>3.5$, which means that at such high supersaturations the dimer has a higher chance to grow to a large supernucleus than to decay to a monomer. Since the condition $P_{2}=1 / 2$ determines approximately the size $n^{*}$ of the nucleus, ${ }^{5}$ Fig. 1 reveals that at $s \approx 3.5$ the dimer is the nucleus. Accordingly, for greater $s$ values the dimer is already a supernucleus. We note also that in the determination of the nucleation rate it is physically meaningful to use only the $P_{2}$ data for $s<4.3$, because this is the value (at $\omega=2$ ) of the spinodal supersaturation $s_{s}$ which limits the $2 \mathrm{D}$ nucleation on the crystal face [4.3 is the $s$ value at the spinodal point of the mean-field adsorption isotherm of a monolayer on the (100) face of Kossel crystal at $\omega=2$; see, e.g., Ref. 16]. Figure 1 shows that, as expected on the basis of general considerations, ${ }^{17} P_{2}$ has no singularity at or near the mean-field spinodal supersaturation $s_{s}=4.3$.

The circles in Fig. 2 represent the nucleation rate $J$ obtained from Eq. (18) with the help of the $P_{2}(s)$ data from Fig. 1. As seen, the present method of the determination of $J$ allows following the change of $J$ with $s$ over nearly 11 orders of magnitude. The standard way to directly determine $J$ by counting the number of supernuclei appearing per unit time does not cover such a wide range of $J$ values. This is evident in Fig. 2 where the squares are the $J(s)$ data obtained by Weeks and Gilmer ${ }^{15}$ in this way. Figure 2 shows also that the present method for the determination of $J$ is in very good agreement with the standard one. We note as well that the lowest $s$ values in Fig. 2 compare with those used by Bostanov et al. ${ }^{18}$ in studying experimentally the 2D nucleation rate in electrochemical growth of the (100) face of a silver crystal (these authors experimented at $s=0.62-0.80$ ).

We can now verify whether the classical nucleation theory describes adequately the $J(s)$ data in Fig. 2. According to this theory, ${ }^{2} J$ is given by Eq. (7) with

$$
A=z f_{n} * C_{0},
$$

where $f_{n^{*}}$ is the attachment frequency of monomers to the nucleus, $C_{0}=1 / a_{0}$ is the concentration of nucleation sites on the crystal face (because the face is free of nucleation-active 
centers), and the Zeldovich factor $z$ is of the form

$$
z=\left(W^{*} / 4 \pi k T n^{* 2}\right)^{1 / 2} .
$$

As this theory treats the $2 \mathrm{D}$ nucleus as a square prism with monomolecular thickness and size-independent specific edge energy $\kappa(\mathrm{J} / \mathrm{m})$, the nucleation work $W^{*}$ and the nucleus size $n^{*}$ are given by $^{2}$

$$
\begin{aligned}
& W^{*}=4 \varepsilon^{2} k T / s, \\
& n^{*}=4 \varepsilon^{2} / s^{2},
\end{aligned}
$$

where

$$
\varepsilon=\kappa a_{0}^{1 / 2} / k T
$$

is the nucleus dimensionless specific edge energy. Also, the square nucleus has $4 n^{* 1 / 2}$ attachment sites which provide nearest-neighbor binding at its periphery so that, in view of Eq. (13), $f_{n^{*}}$ is of the form

$$
f_{n^{*}}=4 n^{* 1 / 2} k_{e} e^{s} .
$$

Combining Eqs. (7), (19)-(22), and (24) yields the classical formula

$$
J=\left(2 / \pi^{1 / 2}\right)\left(k_{e} / a_{0}\right) s^{1 / 2} e^{s} \exp \left(-4 \varepsilon^{2} / s\right)
$$

in which the factor $2 / \pi^{1 / 2}$ corrects the factor $\pi^{1 / 2} / 2$ used by Weeks and Gilmer. ${ }^{15}$ Because of the square shape of the $2 \mathrm{D}$ nucleus, in Eqs. (21), (22), and (25) $\varepsilon$ is related with $\omega$ by the expression ${ }^{19}$

$$
\varepsilon=\omega+\ln \left[\left(1-e^{-\omega}\right) /\left(1+e^{-\omega}\right)\right],
$$

which gives the specific edge energy of an infinitely long (10) step on the (100) face of the Kossel crystal. Thus, Eqs. (21), (22), and (25) represent the $s$ dependence of $W^{*} / k T, n^{*}$, and the dimensionless 2D nucleation rate $J a_{0} / k_{e}$ without free parameters.

Equation (21) does not satisfy the thermodynamic requirement for the annulment of the nucleation work $W^{*}$ at the spinodal supersaturation. Recently, it was shown ${ }^{2,16,20-24}$ that in various cases of nucleation this annulment can be accounted for by the introduction of a correction factor in the corresponding classical formula for $W^{*}$. For the case of 2D nucleation considered here, the correction factor is of the form $\left(1-s^{2} / s_{s}^{2}\right)$ (see Ref. 16) and appears in the right-hand side of Eq. (21). Due to Eq. (7), the nucleation rate is also affected by this correction so that Eq. (25) becomes ${ }^{16}$

$$
J=\left(2 / \pi^{1 / 2}\right)\left(k_{e} / a_{0}\right) s^{1 / 2} e^{s} \exp \left[-\left(4 \varepsilon^{2} / s\right)\left(1-s^{2} / s_{s}^{2}\right)\right] .
$$

The dashed curve in Fig. 2 displays the $J(s)$ dependence from Eq. (25) with $\varepsilon=1.73$, the value following from Eq. (26) at $\omega=2$. We observe that the classical nucleation theory overestimates by an $s$-dependent factor the nucleation rate determined by the simulations. The disagreement is greater than that found by Weeks and Gilmer, ${ }^{15}$ because in Eq. (25) these authors used $\varepsilon=\omega$ which is the low-temperature approximation for $\varepsilon$ following from Eq. (26) for $\omega \gg 1$ [physically, this approximation corresponds to neglecting the molecular roughness of the (10) step at $T>0$ ]. The corrected $J(s)$ dependence from Eq. (27) with spinodal supersaturation $s_{s}=4.3$ is illustrated by the solid curve in Fig. 2. As seen,

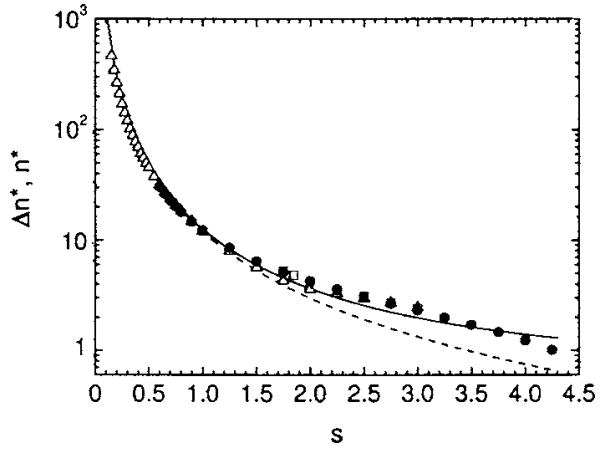

FIG. 3. Supersaturation dependence of the nucleus size: circles-simulation $\Delta n^{*}(s)$ data obtained from Eq. (28); squares- $\Delta n^{*}(s)$ data from Ref. 2, which follow from the simulation $J(s)$ dependence of Weeks and Gilmer; ${ }^{15}$ triangles $-n^{*}(s)$ data of ter Horst and Jansens ${ }^{13}$ obtained by simulations of the cluster growth probability; dashed curve-Gibbs-Thomson Eq. (22); solid curve-Eq. (30).

quantitatively, the description of the simulation data by Eq. (27) is not better than that by Eq. (25). Qualitatively, however, the description is improved, because $J$ from Eq. (27) is greater than $J$ from the simulations by a practically $s$-independent factor. This improvement is visualized by the dotted curve in Fig. 2, which exhibits the $J$ values calculated from Eq. (27) and multiplied by $1 / 40$. Why does this factor improve the performance of Eq. (27) remains an open question.

We can now use Eq. (10) to determine the size of the 2D nucleus with the help of the $P_{2}(s)$ dependence obtained by the simulations. According to Eq. (25), for the kinetic factor $A$ we have $\left(2 / \pi^{1 / 2}\right)\left(k_{e} / a_{0}\right) s^{1 / 2} e^{s}$ so that, in view of Eqs. (14), (16), and (17), for the simulation model Eq. (10) takes the form

$$
\Delta n^{*}=d\left(\ln P_{2}\right) / d s+1-1 / 2 s
$$

which gives $\Delta n^{*}$ more accurately than Eq. (11). The derivative in Eq. (28) can be evaluated with the aid of the function

$$
\ln P_{2}=-10.2127 / s-1.6202+2.1843 s-0.3083 s^{2}
$$

providing the best fit to the simulation data in Fig. 1 and depicted by the curve in this figure. With the so-evaluated derivative, Eq. (28) yields the $\Delta n^{*}(s)$ dependence represented by the circles in Fig. 3. For comparison, the squares in Fig. 3 illustrate the $\Delta n^{*}$ values obtained elsewhere ${ }^{2}$ from the $J(s)$ data of Weeks and Gilmer, ${ }^{15}$ and the triangles show the $n^{*}(s)$ values determined by ter Horst and Jansens ${ }^{13}$ by simulations of the cluster growth probability at $\omega=2$. As seen, despite that the number $n^{*}$ of molecules in the 2D nucleus depends on the cluster definition used by the latter authors, it is close to the definition-independent excess number $\Delta n^{*}$ of molecules in the nucleus. Also, the nucleus size at the lower supersaturations in Fig. 3 is comparable with that operative in real experiments: $n^{*}=19-32$ was found ${ }^{2}$ to correspond to the $s$ range used in the experiments of Bostanov et al. ${ }^{18}$ on 2D nucleation in electrochemical growth of the (100) face of a silver crystal.

Recently, it was shown ${ }^{16}$ that for 2D nucleation in crystal growth the $\Delta n^{*}(s)$ dependence can be expressed approximately as 


$$
\Delta n^{*}=n^{*}(s)\left(1+s^{2} / s_{s}^{2}\right),
$$

where $n^{*}(s)$ is given by the Gibbs-Thomson Eq. (22). The solid curve in Fig. 3 exhibits the $\Delta n^{*}(s)$ dependence predicted by Eq. (30) with $n^{*}$ from Eq. (22) and $s_{s}=4.3$. For comparison, the dashed curve displays the nucleus size $n^{*}$ from Eq. (22). As seen, Eq. (30) provides a better description of the simulation data than the Gibbs-Thomson Eq. (22). Except for $s=4.25$, the $\Delta n^{*}$ values from Eq. (30) are within $17 \%$ of those from the simulations.

\section{CONCLUSION}

In the scope of the Szilard model, the stationary rate $J$ of one-component nucleation has a simple probabilistic interpretation: according to the exact Eq. (6), for any kind of nucleation $J$ is the product of the frequency $f_{1} C_{1}$ with which monomers become dimers and the probability $P_{2}$ that the dimers grow to macroscopically large clusters before decaying to monomers. For that reason, when the supersaturation $s$ is varied at constant temperature, available $P_{2}(s)$ data allow a model-independent evaluation of the nucleus size with the aid of Eqs. (10) or (11).

The $P_{2}(s)$ dependence itself is relatively easily accessible by computer simulations of nucleation. Obtaining this dependence in such a way and using it in Eq. (6) makes it possible to determine $J$ in a rather wide $s$ range. This new method of direct determination of $J$ has the advantage to be independent of the cluster definition employed in the simulations.

The performed kinetic Monte Carlo simulations of 2D nucleation of monolayers on the (100) face of Kossel crystal demonstrate the potentialities of the new method: $J$ is determined over nearly 11 orders of magnitude and the corresponding 2D nucleus is constituted of about 1 to $30 \mathrm{~mol}-$ ecules. The classical nucleation theory overestimates the simulation nucleation rate by an $s$-dependent factor, and a recent corrected formula for $J$, Eq. (27), improves only qualitatively the classical one, Eq. (25). Though describing rather well the simulation findings for the nucleus size, the classical Gibbs-Thomson Eq. (22) is less successful than its corrected version, Eq. (30).

\section{ACKNOWLEDGMENT}

The authors gratefully acknowledge NWO (The Netherlands Organization for Scientific Research) for a grant enabling one of the authors (D.K.) to visit the Delft University of Technology and take part in the present work.

${ }^{1}$ F. F. Abraham, Homogeneous Nucleation Theory (Academic, New York, 1974).

${ }^{2}$ D. Kashchiev, Nucleation: Basic Theory with Applications (ButterworthHeinemann, Oxford, 2000).

${ }^{3}$ R. Becker and W. Döring, Ann. Phys. 24, 719 (1935).

${ }^{4}$ G. M. White, J. Chem. Phys. 50, 4672 (1969).

${ }^{5}$ J. H. ter Horst and D. Kashchiev, J. Chem. Phys. 119, 2241 (2003).

${ }^{6}$ S. Karlin, A First Course in Stochastic Processes (Academic, New York, 1966), p. 205.

${ }^{7}$ T. L. Hill, J. Chem. Phys. 36, 3182 (1962).

${ }^{8}$ T. L. Hill, Thermodynamics of Small Systems (Dover, New York, 1994), Pt. II, p. 167.

${ }^{9}$ D. Kashchiev, J. Chem. Phys. 76, 5098 (1982).

${ }^{10}$ Y. Viisanen, R. Strey, and H. Reiss, J. Chem. Phys. 99, 4680 (1993).

${ }^{11}$ D. W. Oxtoby and D. Kashchiev, J. Chem. Phys. 100, 7665 (1994).

${ }^{12}$ M. Volmer and A. Weber, Z. Phys. Chem., Stoechiom. Verwandtschaftsl. 119, 277 (1926).

${ }^{13}$ J. H. ter Horst and P. J. Jansens, Surf. Sci. 574, 77 (2005).

${ }^{14}$ D. Kashchiev, J. P. van der Eerden, and C. van Leeuwen, J. Cryst. Growth 40, 47 (1977).

${ }^{15}$ J. D. Weeks and G. H. Gilmer, Adv. Chem. Phys. 40, 157 (1979).

${ }^{16}$ D. Kashchiev, J. Cryst. Growth 267, 685 (2004).

${ }^{17} \mathrm{~K}$. Binder, in Phase Transformations in Materials, edited by G. Kostorz (Wiley, New York, 2001), p. 409.

${ }^{18}$ V. Bostanov, W. Obretenov, G. Staikov, and E. Budevski, J. Electroanal. Chem. Interfacial Electrochem. 146, 303 (1983).

${ }^{19}$ H. J. Leamy, G. H. Gilmer, and K. A. Jackson, in Surface Physics of Materials, edited by J. B. Blakeley (Academic, New York, 1975), Vol. I, p. 121

${ }^{20}$ V. Talanquer, J. Chem. Phys. 106, 9957 (1997).

${ }^{21}$ D. Kashchiev, J. Chem. Phys. 118, 1837 (2003)

${ }^{22}$ I. Kusaka, J. Chem. Phys. 119, 1808 (2003).

${ }^{23}$ D. Kashchiev, J. Chem. Phys. 120, 3749 (2004).

${ }^{24}$ V. I. Kalikmanov, J. Chem. Phys. 121, 8916 (2004). 